\title{
DECIMAL CLASSIFICATION FOR AN ANAESTHETIC FILING SYSTEM:
}

\section{A REVISION}

\author{
J.P. BRunelle, M.D. ${ }^{*}$ \\ "Those who cannot remember the past \\ are condemned to repeat it" \\ Santanaya
}

A decimal Classification for an anaesthetic filing system was published in this Journal in $1960 .{ }^{1}$ For its users, it is proving simple, effective and capable of expansion. It seems reasonable to re-issue it with a renovated nomenclature and a much more simple numbering.

Referring to these tables, a number is given to the article to be classified. Any secretary can easily write this number at the upper left corner of a $4 \times 6$ inch filing card with the corresponding reference. All filing cards are set out in numerical order in a small steel filing cabinet.

The number for the present article is 011 .

$\begin{array}{ll}000 & \text { GENERAL CONSIDERATIONS } \\ 010 & \text { BIBLIOGRAPHY } \\ 011 & \text { Information systems } \\ 012 & \text { Anaesthetic journals } \\ 013 & \text { General works on anaesthesia } \\ 019 & \text { Others ... } \\ 020 & \text { PRACTICE OF ANAESTHESIA } \\ 021 & \text { In some centers or countries } \\ 022 & \text { In major disasters } \\ 023 & \text { Group or private practice } \\ 024 & \text { Departments organisation } \\ 025 & \text { Occupational hazards } \\ 026 & \text { Manpower in anaesthesia } \\ 029 & \text { Others ... } \\ 030 & \text { OPERATING ROOMS AND } \\ & \text { OTHERS } \\ 031 & \text { The operating area } \\ 032 & \text { Anesthetic room } \\ 033 & \text { Delivery room } \\ 034 & \text { Recovery room } \\ 035 & \text { Intensive care units (860) } \\ 039 & \text { Others ... } \\ 040 & \text { MEDICO-LEGAL ASPECTS } \\ 050 & \text { ECONOMIC ASPECTS } \\ 060 & \text { TEACHING } \\ 070 & \text { BASIC SCIENCES } \\ 071 & \text { Anatomy } \\ 072 & \text { Physics } \\ 073 & \text { Biochemistry } \\ & \text { Physiology (100) } \\ 079 & \text { Others ... } \\ 0 & \end{array}$

000 GENERALITES

010 BIBLIOGRAPHIE

011 Systèmes d'information

012 Revues d'anesthésie

013 Ouvrages généraux sur l'anesthésie

019 Autres ...

020 PRATIQUEE DE L'ANESTHESIE

021 En certains centres ou pays

022 En temps de catastrophes

023 Pratique de groupe ou privée

024 Organisation des départements

025 Maladies professionnelles

026 La main-d'œuvre en anesthésie

029 Autres ...

0.30 SALLES DE CHIRURGIE ET AUTRES

031 Le bloc opératoire

032 La salle d'induction

033 La salle d'accouchement

034 La salle de réveil

035 Unités de soins intensifs (860)

039 Autres ...

040 ASPECTS MEDICO-LEGAUX

050 ASPECTS ECONOMIQUES

060 ENSEIGNEMENT

070 SCIENCES DE BASE

071 Anatomie

072 Physique

073 Biochimie

Physiologie (100)

Pharmacologie (200)

079 Autres ...

*From the Department of Anesthesia, Notre-Dame Hospital, Montreal, P.Q. 
080 RESEARCHES

081 Experimental techniques

082 Legal aspects

083 Veterinary anaesthesia

089 Others ...

090 OTHERS ...

091 HISTORY OF ANAESTHESIA

092 BIOGRAPHIES

093 SOCIETIES, MUSEUMS

094 TERMINOLOGY

095 ETHICS, RELATIONS

096 ANAESTHETIC RECORDS

097 STATISTICS, COMPUTERS

098 SCIENTIFIC MEETINGS

099 OTHERS ...

100 PHYSIOLOGY

110 THE NERVOUS SYSTEM

111 Transmission of nerve impulses

112 Cerebral cortex (EEG) (942)

113 Reticular formation

114 Pain mechanisms

115 Neuromuscular transmission

116 The autonomic nervous system

119 Others ...

120 THE HEART

121 Physiology of cardiac muscle

122 Excitatory and conductive systems

123 The cardiac cycle

124 Regulation of cardiac function

125 ECG (941)

129 Others ...

130 THE CIRCULATION

131 Haemodynamics

132 Systemic circulation

133 Pulmonary circulatory system

134 Blood flow through special areas

135 Cardiac output, venous return

139 Others ...

140 THE RESPIRATION

141 Pulmonary ventilation

142 Gaseous exchanges

143 Gas transport

144 Regulation of respiration

149 Others ...

150 BODY FLUIDS AND KIDNEYS

151 Acid-base balance

152 Renal physiology

153 Special fluid systems

159 Others ...

160 BLOOD AND IMMUNITY

161 Blood groups

162 Blood coagulation

163 Immunity and allergy

169 Others ...

170 METABOLISM, NUTRITION

171 Bioenergetics
080 RECHERCHES

081 Techniques expérimentales

082 Aspects légaux

083 Anesthésie vétérinaire

089 Autres ...

090 AUTRES ...

091 HISTOIRE DE L'ANESTHESIE

092 BIOGRAPHIES

093 SOCIETES, MUSEES

094 TERMINOLOGIE

095 ETHIQUE, RELATIONS

096 FICHES D'ANESTHESIE

097 STATISTIQUES, ORDINATEURS

098 CONGRES SCIENTIFIQUES

099 AUTRES ...

100 PHYSIOLOGIE

110 LE SYSTEME NERVEUX

111 Transmission de l'influx nerveux

112 Le cortex cérébral (EEG) (942)

113 La formation réticulée

114 Physiologie de la douleur

115 Transmission neuromusculaire

116 Le système nerveux autonome

119 Autres ...

120 LE COEUR

121 Physiologie du muscle cardiaque

122 Zones d'impulsion, de conduction

123 Le cycle cardiaque

124 Régulation de la fonction cardiaque

125 ECG (941)

129 Autres ...

130 LA CIRCULATION

131 Hémodynamique

132 La circulation systémique

133 Système circulatoire pulmonaire

134 Circulations locales

135 Débit cardiaque, retour veineux

139 Autres ...

140 LA RESPIRATION

141 La ventilation pulmonaire

142 Echanges gazeux

143 Transport des gaz

144 Régulation de la respiration

149 Autres ...

150 EAU, ELECTROLYTES, REINS

151 Equilibre acido-basique

152 Physiologie rénale

153 Compartiments hydriques

159 Autres ...

160 LE SANG ET L'IMMUNITE

161 Groupes sanguins

162 Coagulation sanguine

163 Immunité et allergie

169 Autres ...

170 METABOLISME, NUTRITION

171 Bioénergétique 
172 Temperature regulation

173 Functions of the liver

179 Others ...

180 ENDOCRINOLOGY

181 Hypophyseal hormones

182 Adrenocortical hormones

183 Thyroid hormones

189 Others ...

190 OTHERS ...

200 PHARMACOLOGY

210 INHALATION ANAESTHETIC AGENTS (340)

211 Halothane

212 Methoxyflurane

213 Forane

214 Nitrous oxide

219 Others ...

220 INTRAVENOUS ANAESTHETICS (370)

221 Barbiturates (thiopentone ..)

222 Steroids (hydroxydione ..)

223 Dissociative (agents kétamine . .)

224 Phenoxyacetamides (propanidid ..)

225 Hydroxybutyrate (Gamma-OH)

229 Others ...

230 LOCAL ANAESTHETICS (410)

231 Lignocaine (Xylocaine)

232 Mepivacaine (Carbocain)

233 Bupivacaine (Marcain)

239 Others ...

240 OPIATES AND SUBSTITUTES

241 Morphine

242 Codeine

243 Dihydromorphinone (Dilaudid)

244 Meperidine (Demerol)

245 Fentanyl (Sublimaze)

246 Pentazocine (Talwin)

249 Others ...

250 NARCOTIC ANTAGONISTS

251 Levallorphan (Lorfan)

252 Nalorphine (Nalline)

259 Others ...

260 NEUROLEPTICS

261 Droperidol (Inapsin)

262 Diazepam (Valium)

263 Promethazine (Phenergan)

269 Others ...

270 NEUROMUSCULAR BLOCKING DRUGS (960)

271 d-tubocurarine

272 Gallamine (Flaxedil)

273 Suxamethonium (Succinylcholine)

274 Steroids (Pancuronium ...)

279 Others ...
172 Régulation thermique

173 Physiologie hépatique

179 Autres ...

180 ENDOCRINOLOGY

181 Hormones hypophysaires

182 Hormones surrénaliennes

183 Hormones thyroidiennes

189 Autres ...

190 AUTRES ...

200 PHARMACOLOGIE

210 ANESTHESIQUES PAR INHALATION (340)

211 Halothane

212 Methoxyflurane

213 Forane

214 Protoxyde d'azote

219 Autres ...

220 ANESTHESIQUES INTRAVEINEUX (370)

221 Barbituriques (thiopental . .)

222 Stéroides (hydroxydione ..)

223 Agents de dissiciation (kétamine ..)

224 Phenoxyacetamides (propanidid ..)

225 Hydroxybutyrate (Gamma-OH)

229 Autres ...

230 ANESTHESIQUES LOCAUX (410)

231 Lignocaine (Xylocaine)

232 Mepivacaine (Carbocaine)

233 Bupivacaine (Marcain)

239 Autres ...

240 OPIACES ET SUCCEDANES

241 Morphine

242 Codéine

243 Dihydromorphinone (Dilaudid)

244 Meperidine (Démerol)

245 Fentanyl (Sublimaze)

246 Pentazocaine (Talwin)

249 Autres ...

250 ANTIDOTES DES NARCOTIQUES

251 Levallorphan (Lorfan)

252 Nalorphine (Nalline)

259 Autres ...

260 NEUROLEPTIQUES

261 Dropéridol (Inapsin)

262 Diazépam (Valium)

263 Prométhazine (Phénergan)

269 Autres ...

270 MYORESOLUTIFS (960)

271 d-tubocurarine

272 Gallamine (Flaxédil)

273 Suxaméthonium (Succinylcholine)

274 Stéroides (Pancuronium . .)

279 Autres ... 
BRUNELLE: DECIMAL CLASSIFICATION

280 AUTONOMIC NERVOUS SYSTEM DRUGS

281 Parasympathomimetics

282 Parasympatholytics

283 Sympathomimetics

284 Sympatholytics

285 Ganglionic blocking agents

289 Others ...

290 OTHERS ...

291 ANALEPTICS (847)

292 HYPNOTICS

293 CARDIAC DRUGS

294 HISTAMINE, ANTIHISTAMINICS

295 ANTIEMETICS

296 DIURETICS

297 LOCALLY ACTING DRUGS

298 ANTICOAGULANTS

299 OTHERS ...

300 GENERAL ANAESTHESIA

310 MECHANISMS OF GENERAL ANAESTHESIA

320 DEPTH OF ANAESTHESIA

330 INHALATION ANAESTHESIA

331 Equipment

332 Anaesthesia apparatus

$333 \mathrm{CO}_{2}$ absorption

334 Humidification (855)

335 Denitrogenation

336 Dead-space

337 Disposing of exhaust gases

338 Complications

339 Others ...

340 INHALATION ANAESTHETIC AGENTS (210)

341 Solubility

342 Metabolism

343 Synergism

344 Anaesthetic potency

349 Others ...

350 METHODS

351 Semiclosed circuits

352 Closed circuits

353 T-piece technics

359 Others ...

360 ARTIFICIAL AIRWAY

361 Oropharyngeal airway

362 Nasopharyngeal airway

363 Orotracheal intubation

364 Nasotracheal intubation

365 Endobronchial intubation

366 Blind intubation

367 Prolonged intubation

368 Complications

369 Others ...
280 DROGUES DU SYSTEME NERVEUX AUTONOME

281 Parasympathomimétiques

282 Parasympatholytiques

283 Sympathomimétiques

284 Sympatholytiques

285 Ganglioplégiques

289 Autres ...

290 AUTRES ..

291 ANALEPTIQUES (847)

292 HYPNOTIQUES

293 DROGUES CARDIAQUES

294 HISTAMINE, ANTIHISTAMINIQUES

295 ANTIEMETIQUES

296 DIURETIQUES

297 DROGUES D'ACTION LOCALE

298 ANTICOAGULANTS

299 AUTRES ...

300 ANESTHESIE GENERALE

310 MECANISMES DE L'ANESTHESIE

320 PROFONDEUR DE L'ANESTHESIE

330 ANESTHESIE PAR INHALATION

331 Equipement

332 Appareils à anesthésie

333 Absorption du $\mathrm{CO}_{2}$

334 Humidification (855)

335 Elimination de l'azote

336 Espace mort

337 Elimination des gaz éxalés

338 Complicetions

339 Autres ...

340 ANESTHESIQUES PAR INHALATION (210)

341 Solubilité

342 Métabolisme

343 Synergisme

344 Puissance anesthésique

349 Autres ...

350 METHODES

351 Circuits semi-fermés

352 Circuits fermés

353 Techniques du tube en $\mathbf{T}$

359 Autres ...

360 VOIES RESPIRATOIRES ARTI-

\section{FICIELLES}

361 Oropharyngée

362 Nasopharyngée

363 Intubation orotrachéale

364 Intubation nasotrachéale

365 Intubation endobronchique

366 Intubation à l'aveugle

367 Intubation prolongée

368 Complications

369 Others ... 
370 INTRAVENOUS ANAESTHESIA (220)

371 Induction agents

372 Emulsions

373 Local anaesthetic agents

374 Opiates and substitutes

375 Neuroleptics

376 Neuroleptanalgesia

377 Clinical studies

378 Complications

379 Others ...

380 COMPLICATIONS

390 OTHERS ...

391 PEDIATRIC GENERAL ANAESTHESIA

392 MULTIPLE GENERAL ANAESTHESIA

399 OTHERS ...

400 REGIONAL ANAESTHESIA

410 LOCAL ANAESTHETICS (230)

411 Pharmacology

412 Comparative pharmacology

413 Toxicity

414 Carbonated local anaesthetics

419 Others ...

420 ADJUNCTS TO LOCAL ANAESTHETICS

421 Vasoconstrictors

422 Neurolytic agents

429 Others ...

430 BLOCKING THE PERIPHERAL NERVES

431 Intercostal nerve block

432 Paravertebral nerve block

433 Femoral nerve block

434 Obturator nerve block

435 Sciatic nerve block

436 Pudental nerves block

439 Others ...

440 PLEXUS BLOCKS

441 Cervical plexus block

442 Brachial plexus block

449 Others ...

450 AUTONOMIC NERVOUS SYSTEM BLOCKS

451 Stellate block

452 Laryngeal nerve block

453 Lumbar sympathetic nerves block

459 Others ...

460 SUBARACHNOID BLOCK

470 PERIDURAL BLOCK

471 Ordinary technique

472 Continuous peridural block

473 Caudal block

479 Others ...
370 ANESTHESIE INTRA-

VEINEUSE (220)

371 Agents d'induction

372 Emulsions

373 Anesthésiques locaux

374 Opiacés et succédanés

375 Neuroleptiques

376 Neuroleptanalgésie

377 Etudes cliniques

378 Complications

379 Autres ...

380 COMPLICATIONS

390 AUTRES ...

391 ANESTHESIE GENERALE EN PEDIATRE

392 ANESTHESIES GENERALES MULTIPLES

399 AUTRES ...

400 ANESTHESIE REGIONALE

410 ANESTHESIQUES LOCAUX (230)

411 Pharmacologie

412 Pharmacologie comparée

413 Toxicité

414 Anesthésiques locaux carbonatés

419 Autres ...

420 DROGUES ADJUVANTES

421 Vasoconstricteurs

422 Agents neurolytiques

429 Autres ...

430 BLOC DES NERFS PERIPHERIQUES

431 Nerfs intercostaux

432 Nerfs paravertébraux

433 Nerf fémoral

434 Nerf obturateur

435 Nerf sciatique

436 Bloc honteux

439 Autres ...

440 BLOC DES PLEXUS

441 Bloc du plexus cervical

442 Bloc du plexus brachial

449 Autres ...

450 BLOCS DU SYSTEME NERVEUX AUTONOME

451 Bloc stellaire

452 Bloc laryngé

453 Bloc sympathique lombaire

459 Autres ...

460 BLOC SOUS-ARACHNOIDIEN

470 BLOC PERIDURAL

471 Technique ordinaire

472 Bloc péridural continu

473 Bloc caudal

479 Autres... 
BRUNELLE: DECIMAL CLASSIFICATION

480 COMPLICATIONS

490 OTHERS...

491 CRANIAL NERVES BLOCK

492 LOCAL AND TOPICAL ANAESTHESIA

493 REGIONAL INTRAVENOUS ANAESTHESIA

494 DIAGNOSTIC NERVE BLOCKS

495 THERAPEUTIC NERVE BLOCKS

499 OTHERS ...

500 PRE-ANAESTHETIC CONDITION

510 CARDIO-VASCULAR DISEASES

511 Heart diseases

512 Hypertension

519 Others ...

520 PULMONARY DISEASES

521 Bronchitis, asthma, emphysema

522 Cystic fibrosis

529 Others ...

530 NERVOÜS SYSTEM DISORDERS

531 Brain and spinal cord lesions

532 Familial dysautonomia

533 Neuroblastomas

539 Others ...

540 NEUROMUSCULAR DISORDERS

541 Myasthenia

542 Muscular dystrophy

543 Multiple sclerosis

549 Others ...

550 METABOLIC DISORDERS

551 Obesity

552 Porphyria

553 Febrile patients

554 Cystinuria

559 Others ...

560 ENDOCRINE DISTURBANCES

561 Pituitary gland

562 Thyroid gland

563 Adrenal glands

564 Pancreas (Diabetes)

569 Others ...

570 PATIENTS ON THERAPY (770)

571 Steroid therapy

572 Digitalis, quinidine . .

573 Hypotensive drugs

574 Neuroleptics

575 Antibiotics

576 Monoamine oxydase inhibitors

577 Anti-cancer drugs

578 Levodopa
480 COMPLICATIONS

490 AUTRES

491 BLOC DES NERFS CRANIENS

492 ANESTHESIE LOCALE, DE SURFACE

493 REGIONALE INTRA-VEINEUSE

494 BLOCS DIAGNOSTIQUES

495 BLOCS THERAPEUTIQUES

499 AUTRES ...

500 CONDITION PRE-ANESTHESIQUE

510 MALADIES CARDIO-VASCULAIRES

511 Cardiopathies

512 Hypertension artérielle

519 Autres ...

520 MALADIES PULMONAIRES

521 Bronchite, asthme, emphysème

522 Maladie fibro-kystique

529 Autres ...

530 TROUBLES DU SYSTEME NERVEUX

531 Lésions cérébrales, médullaires

532 Dysautonomie familiale

533 Neuroblastomes

539 Autres ...

540 TROUBLES NEUROMUSCU-

\section{LAIRES}

541 Myasthénie

542 Dystrophie musculaire

543 Sclérose en plaques

549 Autres ...

550 TROUBLES METABOLIQUES

551 Obésité

552 Porphyrie

553 Patients fébriles

554 Cystinurie

559 Autres ...

560 TROUBLES ENDOCRINIENS

561 Hypophyse

562 Thyroide

563 Surrénales

564 Pancréas (Diabète)

569 Others ...

570 PATIENTS SOUS THERAPIE (770)

571 Stéroides

572 Digitale, quinidine . .

573 Hypotenseurs

574 Neuroleptiques

575 Antibiotiques

576 Inhibiteurs-monoamine oxydase

577 Drogues anti-cancéreuses

578 Lévodopa 
579 Others ...

580 MISCELLANEOUS

581 Patients with "full stomach" (723)

582 Patients in shock (815)

583 Drug abusers (817)

584 Pregnant women (795)

585 Carcinoid tumors

586 Cholinesterase disorders (962)

587 Burned, traumatized patients

588 Intestinal obstruction

589 Others ...

590 OTHERS ...

591 SKIN DISORDERS

592 BLOOD DISTURBANCES

593 RENAL DISEASES (750)

594 HEPATIC DISEASES (760)

595 UNCOMMON SYNDROMES

599 OTHERS ...

600 SPECIAL ANAESTHETIC PROCEDURES

610 PEDIATRIC ANAESTHESIA

611 Anatomic, physiologic aspects

612 Pre-anaesthetic medication

613 Pre-anaesthetic condition

614 Special operative procedures

615 Fluid therapy

616 Mechanical ventilation

617 Resuscitation, inhalotherapy

618 Complications

619 Others ...

620 GERIATRIC ANAESTHESIA

630 OBSTETRICAL ANAESTHESIA

631 Maternal and foetal physiology

632 Pre-anaesthetic condition

633 Placental transmission of drugs

634 Anaesthesia for Caesarian section

635 Regional anaesthesia

636 Resuscitation of the new-born

637 Maternal and foetal mortality

638 Complications

639 Others ...

640 DENTAL ANAESTHESIA

650 NON-SURGICAL ANAESTHESIA

651 Electroconvulsive therapy

652 Cardioversions

653 Endoscopy

654 Cardiac catheterization

655 Pneumoencephalography

656 Angiography

659 Others ...

660 ANAESTHESIA-REGIONAL SURGERY

661 Head and neck surgery
579 Autres ...

580 DIVERS

581 Patients non à jeun (723)

582 Patients en état de choc (815)

583 Toxicomanes (817)

584 Patientes gravides (795)

585 Tumeurs carcinoides

586 Troubles de la cholinestérase (962)

587 Brûlures, traumatismes graves

588 Obstruction intestinale

589 Autres ...

590 AUTRES ...

591 MALADIES DE LA PEAU

592 TROUBLES HEMATIQUES

593 MALADIES RENALES (750)

594 MALADIES HEPATIQUES (760)

595 SYNDROMES EXCEPTIONNELS

599 AUTRES ...

600 ANESTHESIES SPECIALES

610 ANESTHESIE PEDIATRIQUE

611 Aspects anatomiques, physiologiques

612 Médication pré-anesthésique

613 Condition pré-anesthésique

614 Interventions spéciales

615 Thérapie liquidienne

616 Ventilation mécanique

617 Réanimation, inhalothérapie

618 Complications

619 Autres ...

620 ANESTHESIE GERIATRIQUE

630 ANESTHESIE OBSTETRICALE

631 Physiologie maternelle et foétale

632 Condition pré-anesthésique

63.3 Passage trans-placentaire des drogues

634 Anesthésie pour césarienne

635 Anesthésie régionale

636 Réanimation du nouveau-né

637 Mortalité maternelle et foetale

638 Complications

639 Autres ...

640 ANESTHESIE DENTAIRE

650 ANESTHESIES NON-CHIRURGICALES

651 Thérapie électro-convulsive

652 Cardioversions

653 Endoscopie

654 Cathétérismes cardiaques

655 Pneumoencéphalographie

656 Angiographie

659 Autres ...

660 ANESTHESIE-CHIRURGIE REGIONALE

661 Chirurgie de la tête et du cou 
662 Thoracic surgery

663 Abdominal surgery

664 Ano-rectal surgery

665 Others ...

670 ANAESTHESIA-SURGICAL, SPECIALTIES

671 Neurosurgery

672 Cardiac surgery (980)

673 Lungs surgery

674 Vascular surgery

675 ORL

676 Urologic surgery

677 Plastic surgery

678 Ophtalmologic surgery

679 Others ...

680 ANAESTHESIA-ORGAN TRANSPLANTATIONS

690 AUTRES ...

691 EMERGENCY SURGERY

692 OUT-PATIENT SURGERY

693 SURGERY FOR SEVERE INJURIES

694 CONJOINED TWINS SURGERY

699 OTHERS ...

700 COMPLICATIONS

710 CARDIO-VASCULAR COMPLICATIONS

711 Abnormal rhythms of the heart

712 Fat or air embolism

713 Hypo and hypertension

714 Cardiac arrest (820)

715 Myocardial infarction

719 Others ...

720 RESPIRATORY COMPLICATIONS

721 Laryngospasm, bronchospasm

722 Pulmonary oedema (812)

723 Bronchial aspiration (581)

724 Atelectasis

725 Pulmonary collapse

726 Prolonged apnoea

727 Pneumothorax

728 Hiccup

729 Others ...

730 NEUROLOGICAL COMPLICATIONS

731 Shivering, convulsions

732 Prolonged unconsciousness

733 Mental disturbances

739 Others ...

740 METABOLIC DISTURBANCES

741 Hypo-, hyperthermia

742 Malignant hyperpyrexia

749 Others ...
662 Chirurgie thoracique

663 Chirurgie abdominale

664 Chirurgie ano-rectale

665 Autres ...

670 ANESTH.-SPECIALITES CHIRURGICALES

671 Neurochirurgie

672 Chirurgie cardiaque (980)

673 Chirurgie pulmonaire

674 Chirurgie vasculaire

675 ORL

676 Chirurgie urologique

677 Chirurgie plastique

678 Chirurgie ophtalmologique

679 Autres ...

680 ANESTH.-TRANSPLANTATIONS D'ORGANES

690 AUTRES ...

691 CHIRURGIE D'URGENCE

692 CHIRURGIE AMBULATOIRE

693 GRANDS TRAUMATISMES

694 JUMEAUX CONJOINTS

699 AUTRES ...

700 COMPLICATIONS

710 COMPLICATIONS CARDIO-VASCULAIRES

711 Troubles du rythme cardiaque

712 Embolie graisseuse, gazeuse

713 Hypo et hypertension

714 Arrêt cardiaque (820)

715 Infarctus du myocarde

719 Autres ...

720 COMPLICATIONS RESPIRATOIRES

721 Spasme glottique, bronchique

722 Oédème pulmonaire (812)

723 Aspiration bronchique (581)

724 Atélectasie

725 Collapsus pulmonaire

726 Apnée prolongée

727 Pneumothorax

728 Hoquet

729 Autres ...

730 COMPLICATIONS NEUROLOGIQUES

731 Tremblements, convulsions

732 Inconscience prolongée

733 Troubles mentaux

739 Autres ...

740 TROUBLES METABOLIQUES

741 Hypo, hyperthermie

742 Hyperthermie maligne

749 Autres ... 
750 RENAL COMPLICATIONS (593)

760 HEPATIC COMPLICATIONS (594)

770 DRUGS INTERACTIONS (570)

780 HAZARDS OF ANAESTHETIC EQUIPMENT

781 Fires and explosions

782 Burns

783 Infections

789 Others ...

790 OTHERS ...

791 AWARENESS DURING SURGERY

792 COMPLICATIONS FROM SURGICAL POSTURE

793 HYPERSENSIBILITY REACTIONS

794 MUSCLE PAIN

795 TERATOGENIC EFFECTS (584)

796 ANAESTHETIC DEATHS

799 OTHERS ... (338) (368) (378) (380) (480) (618) (638) (880) (958) (968)

800 RESUSCITATION, INHALOTHERAPY

810 INDICATIONS

811 Asthma, emphysema, bronchitis (521)

812 Pulmonary oedema (722)

813 Fat and air embolism (712)

814 Drowning

815 Shock (582)

816 Tetanus

817 Poisoning (583)

818 Electrocution

819 Others

820 CARDIO-RESPIRATORY RESUSCITATION

830 CARDIO-VASCULAR RESUSCITATION

831 Heart massage

832 Ventricular defibrillation

839 Others ...

840 RESPIRATORY RESUSCITATION

841 Mouth-to-mouth resuscitation

842 Oro, nasotracheal intubation

843 Tracheotomy

844 Bronchoscopy

845 Manual methods

846 Mechanical ventilation

847 Analeptics (291)
750 COMPLICATIONS RENALES (593)

760 COMPLICATIONS HEPATIQUES (594)

770 INTERACTIONS MEDICAMENTEUSES (570)

780 ACCIDENTS DUS A L'EQUIPEMENT

781 Enflammations, explosions

782 Brûlures

783 Infections

789 Autres ...

790 AUTRES

791 CONSCIENCE PEROPERATOIRE

792 COMP.-MALPOSITION CHIRURGICALE

793 REACTIONS D'HYPERSENSIBILITE

794 DOULEURS MUSCULAIRES

795 EFFECTS TERATOGENIQUES (584)

796 MORTALITE ANESTHESIQUE

799 AUTRES ... (338) (368) (378) (380) (480) (618) (638) (880) (958) (968)

800 REANIMATION, INHALOTHERAPIE

810 INDICATIONS

811 Asthme, emphysème, bronchite (521)

812 Oédème pulmonaire (722)

813 Embolie graisseuse, gazeuse (712)

814 Noyade

815 Choc (582)

816 Tétanos

817 Intoxications (583)

818 Electrocution

819 Autres ...

820 REANIMATION CARDIORESPIRATOIRE

830 REANIMATION CARDIOVASCULAIRE

831 Massage cardiaque

832 Défibrillation ventriculaire

839 Autres ...

840 REANIMATION RESPIRATOIRE

841 Bouche à bouche

842 Intubation oro, nasotrachéale

843 Trachéotomie

844 Bronchoscopie

845 Méthodes manuelles

846 Ventilation mécanique

847 Analeptiques (291) 


848 Complications
849 Others ...
850 INHALOTHERAPY
851 Oxygen therapy
852 Hyperbaric oxygen
853 Oxygen toxicity
854 Aerosol therapy
855 Humidification (334)
856 Analysers (O OO $_{2}$ )
859 Others ...
860 INTENSIVE CARE (035)
870 PROGNOSIS, CRITERIA OF
DEATH
880 COMPLICATIONS
890 OTHERS ...
900 OTHERS
910 PREANAESTHETIC PERIOD
911 Preanaesthetic rounds
912 Evaluating the patient
913 Preparing the patient
914 Preanaesthetic medication
919 Others ...
920 POSTANAESTHETIC PERIOD

921 Postanaesthetic rounds

922 Postoperative care

929 Others ...

930 FLUID THERAPY

931 Technique

932 Water, electrolytes

933 Plasma expanders

934 Blood banks

935 Blood volume, blood losses

936 Packed red blood cells

937 Autotransfusions

938 Complications

939 Others ...

940 MONITORING, CLINICAL ANALYSIS

941 ECG (125)

942 EEG (112)

943 Ventilation monitoring

947 Blood gases analysis

944 Blood pressure monitoring

945 Venous pressure monitoring

946 Temperature monitoring

948 Complications

949 Others ...

950 MECHANICAL PULMONARY VENTILATION

951 Mechanical ventilators

952 Norms, evaluating the ventilation

953 IPPB
848 Complications

849 Autres ...

850 INHALOTHERAPIE

851 Oxygénothérapie

852 Oxygène hyperbare

853 Toxicité de l'oxygène

854 Aérosolthérapie

855 Humidification (334)

856 Analyseurs $\left(\mathrm{O}_{2}, \mathrm{CO}_{2}\right)$

859 Autres ...

860 LES SOINS INTENSIFS (035)

870 PRONOSTIC, CRITERES DE LA MORT

880 COMPLICATIONS

890 AUTRES ...

900 AUTRES

910 PERIODE PRE-ANESTHESIQUE

911 Visite pré-opératoire

912 Evaluation du patient

913 Préparation du patient

914 Médication pré-anesthésique

919 Authres ...

920 PERIODE POST-ANESTHESIQUE

921 Visite post-anesthésique

922 Soins post-opératoires

929 Autres ...

930 THERAPIE LIQUIDIENNE

931 Techniques

932 Eau, électrolytes

9.33 Substituts du plasma

934 Banques de sang

935 Volume sanguin, pertes sanguines

936 Globules rouges concentrés

937 Autotransfusions

938 Complications

939 Autres ...

940 MONITORING, ANALYSES CLINIQUES

941 ECG (125)

942 EEG (112)

943 Monitoring de la ventilation

944 Monitoring de la pression artérielle

945 Monitoring de la pression veineuse

946 Monitoring de la température

947 Analyse des gaz sanguins

948 Complications

949 Autres ...

950 VENTILATION PULMONAIRE MECANIQUE

951 Respirateurs mécaniques

952 Normes, mesures de la ventilation

953 RPPI 
954 Hyperventilation

955 Hypoventilation

956 Negative pressure ventilation

957 Prolonged mechanical ventilation

958 Complications

959 Others ...

960 MUSCULAR RELAXATION (270)

961 Types of neuromuscular block

962 Cholinesterase disturbances (586)

963 Mixing of relaxants

964 Measurement of neuromuscular block

965 Reversing neuromuscular block

966 Curarisation of the new-born

967 Regional curarization

968 Complications

969 Others ...

970 EQUIPMENT

971 Care, disinfection, sterilization

972 Hazards of anaesthetic equipment (780)

979 Others ...

980 EXTRACORPOREAL CIRCULATION (672)

990 OTHERS

991 JNDUCED HYPOTHERMIA

992 CONTROLLED HYPOTENSION

993 ELECTRONARCOSIS

994 HYPNOSIS

995 ACUPUNCTURE

999 OTHERS ...
954 Hyperventilation

955 Hypoventilation

956 Ventilation en pression négative

957 Ventilation mécanique prolongée

958 Complications

959 Autres ...

960 RELACHEMENT MUSCULAIRE (270)

961 Types de blocs neuromusculaires

962 Troubles de la cholinestérase (586)

963 Interactions des myorésolutifs

964 Mesures du bloc neuromusculaire

965 Renversement de la curarisation

966 Curarisation du nouveau-né

967 Curarisation régionale

968 Complications

969 Autres ...

970 INSTRUMENTATION

971 Soins, désinfection, stérilisation

972 Accidents tenant aux appareils (780)

979 Autres ...

980 CIRCULATION EXTRACORPORELLE (672)

990 AUTRES

991 HYPOTHERMIE PROVOQUEE

992 HYPOTENSION CONTROLEE

993 ELECTRONARCOSE

994 HYPNOSE

995 ACUPUNCTURE

999 AUTRES ...

Une méthode de classification décimale des ouvrages sur l'anesthésie a été publiée en $1960 .{ }^{1}$ A ceux qui l'utilisent, elle paraît simple, efficace et extensible en tout point. C'est la raison de cette scconde parution dans une forme renouvelée et avec un numérotage très simplifié.

En se reférant à ces tables, il suffit d'inscrire près du titre d'un article à classer, son chiffre correspondant. Une secrétaire n'a plus qu'à inscrire ce chiffre au coin supérieur gauche d'une fiche de $4 \times 6$ pouces, et la référence. Les fiches sont rangées par ordre numérique dans un fichier métallique.

Le numéro du présent article : 011.

\section{REFERENCE}

1. Brunelle, J.P. Decimal classification for an anaesthetic filing system, Canad. Anaesth. Soc. J. 7: 179 (April 1960). 